PROCEEDINGS OF THE

AMERICAN MATHEMATICAL SOCIETY

Volume 133, Number 4, Pages 1143-1150

S 0002-9939(04)07599-9

Article electronically published on October 14, 2004

\title{
A BIG PICARD THEOREM FOR QUASIREGULAR MAPPINGS INTO MANIFOLDS WITH MANY ENDS
}

\author{
ILKKA HOLOPAINEN AND PEKKA PANKKA \\ (Communicated by Juha M. Heinonen)
}

\begin{abstract}
We study quasiregular mappings from a punctured Euclidean ball into $n$-manifolds with many ends and prove, by using Harnack's inequality, a version of the big Picard theorem.
\end{abstract}

\section{INTRODUCTION}

The classical big Picard theorem states that a holomorphic mapping of the punctured unit disc $\{z \in \mathbb{C}: 0<|z|<1\}$ into the complex plane omitting two values has a meromorphic extension to the whole disc; see e.g. A, Theorem 1-14]. In [R1] Rickman proved a counterpart of Picard's theorem for quasiregular mappings (Theorem 1.1) and its local version (Theorem 1.2) corresponding to the big Picard theorem.

Theorem 1.1 ([R1]). For each integer $n \geq 2$ and each $K \geq 1$ there exists a positive integer $q=q(n, K)$ such that if $f: \mathbb{R}^{n} \rightarrow \mathbb{R}^{n} \backslash\left\{a_{1}, \ldots, a_{q}\right\}$ is $K$-quasiregular and $a_{1}, \ldots, a_{q}$ are distinct points in $\mathbb{R}^{n}$, then $f$ is constant.

Theorem $1.2([\mathbb{R} 1])$. Let $G=\left\{x \in \mathbb{R}^{n}:|x|>s\right\}$, and let $f: G \rightarrow \mathbb{R}^{n} \backslash\left\{a_{1}, \ldots, a_{q}\right\}$ be a $K$-quasiregular mapping, where $a_{1}, \ldots, a_{q}$ are distinct points in $\mathbb{R}^{n}$ and $q=$ $q(n, K)$ is the integer in Theorem 1.1. Then the limit $\lim _{|x| \rightarrow \infty} f(x)$ exists.

Recall that a continuous mapping $f: M \rightarrow N$ between smooth, oriented, and connected Riemannian $n$-manifolds $M$ and $N$ is called $K$-quasiregular if it belongs to the Sobolev space $W_{\text {loc }}^{1, n}(M, N)$ and satisfies an inequality

$$
\left\|T_{x} f\right\|^{n} \leq K J(x, f)
$$

for a.e. $x \in M$. Here $\left\|T_{x} f\right\|$ is the operator norm of the tangent map $T_{x} f: T_{x} M \rightarrow$ $T_{f(x)} N$, and $J(x, f)$ is the Jacobian determinant of $f$ at $x$ uniquely defined by $\left(f^{*} \operatorname{vol}_{N}\right)_{x}=J(x, f)\left(\operatorname{vol}_{M}\right)_{x}$ almost everywhere.

In this paper we consider an analogue of the big Picard theorem for quasiregular mappings of the punctured Euclidean unit ball $B^{n} \backslash\{0\}$ into a Riemannian $n$ manifold with sufficiently many ends. Given a compact set $C \subset N$ we say that a component, $E$, of $N \backslash C$ is an end of $N$ with respect to $C$ if the closure $\bar{E}$ is

Received by the editors August 26, 2003 and, in revised form, December 2, 2003.

2000 Mathematics Subject Classification. Primary 30C65.

Key words and phrases. Essential singularity, Harnack inequality, Picard theorem, quasiregular mappings.

Both authors were supported in part by the Academy of Finland, project 53292.

(C)2004 American Mathematical Society 
noncompact. Furthermore, we say that $N$ has at least $q$ ends if $N$ has at least $q$ ends with respect to some compact set $C \subset N$. We say that a mapping $f: B^{n} \backslash\{0\} \rightarrow N$

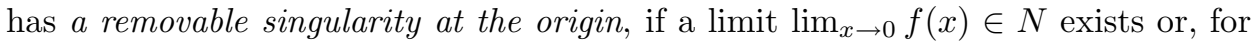
every compact set $C \subset N$, there exists $r \in(0,1)$ such that $f\left(B^{n}(0, r) \backslash\{0\}\right)$ is contained in an end with respect to $C$. Otherwise, we say that $f$ has an essential singularity at the origin.

We can now state our main result, which is a generalization of Theorem 1.2 ,

Theorem 1.3. Let $N$ be a Riemannian $n$-manifold. For every $K \geq 1$ there exists $q=q(K, n)$ such that every $K$-quasiregular mapping $f: B^{n} \backslash\{0\} \rightarrow N$ has a removable singularity at the origin if $N$ has at least $q$ ends.

It is worth noting that the proof of Theorem 1.2 makes use of both of the main tools in the theory of quasiregular mappings: the method of extremal length and the nonlinear potential theory. In particular, relatively sharp estimates for the modulus of certain path families are needed both in the domain and in the range of the mapping, thus making it hard to extend the method beyond the Euclidean setting. In [HR3] Holopainen and Rickman applied a method of Lewis ([L]) that relies on Harnack's inequality to prove the following general version of Picard's theorem; see also [EL], [HR1], and [HR2] for other proofs and versions of Picard's theorem.

Theorem 1.4 ([HR3]). Suppose that a complete Riemannian n-manifold $M$ admits a global Harnack's inequality for positive $\mathcal{A}$-harmonic functions of type $n$. Assume, furthermore, that $M$ has the following covering property: for each $0<k<1$ there exists an integer $m=m(k)$ such that every ball $B(x, r) \subset M$ can contain at most $m$ disjoint balls of radius $k r$. Then, for every $K \geq 1$, there exists a constant $q$ such that every $K$-quasiregular mapping $f: M \rightarrow N$ must be constant if $N$ has at least $q$ ends.

The proof of our main result (Theorem 1.3) is closely related to the proof of Theorem 1.4 Thus our proof relies on Harnack's inequality for positive $\mathcal{A}$-harmonic functions. We recall that a continuous function $u: N \rightarrow \mathbb{R}$ is $\mathcal{A}$-harmonic (of type $p \in(1, \infty))$ if $u \in W_{\mathrm{loc}}^{1, p}(N)$ and satisfies an equation

$$
-\operatorname{div} \mathcal{A}_{x}(\nabla u)=0
$$

weakly, that is,

$$
\int_{N}\left\langle\mathcal{A}_{x}(\nabla u), \nabla \varphi\right\rangle d x=0
$$

for all $\varphi \in C_{0}^{\infty}(N)$. Here $\mathcal{A}: T N \rightarrow T N$ is a map such that $\mathcal{A}_{x}: T_{x} N \rightarrow T_{x} N$ is continuous for a.e. $x \in N$ and the map $x \mapsto \mathcal{A}_{x}(X)$ is measurable for every measurable vector field $X$. We assume that there exist constants $0<\alpha \leq \beta<\infty$ such that

$$
\left\langle\mathcal{A}_{x}(\xi), \xi\right\rangle \geq \alpha|\xi|^{p}
$$

and

$$
\left|\mathcal{A}_{x}(\xi)\right| \leq \beta|\xi|^{p-1}
$$

for a.e. $x \in N$ and for all $\xi \in T_{x} N$. We assume further that for a.e. $x \in N$,

$$
\left\langle\mathcal{A}_{x}(\xi)-\mathcal{A}_{x}(\eta), \xi-\eta\right\rangle>0
$$

whenever $\xi \neq \eta$, and

$$
\mathcal{A}_{x}(\lambda \xi)=|\lambda|^{p-2} \lambda \mathcal{A}_{x}(\xi)
$$


whenever $\lambda \in \mathbb{R} \backslash\{0\}$. In the special case $\mathcal{A}_{x}(\xi)=|\xi|^{p-2} \xi, \mathcal{A}$-harmonic functions are called $p$-harmonic.

In the sequel we use repeatedly the fact that quasiregular mappings are $\mathcal{A}$ harmonic morphisms (see e.g. [HKM] ), especially the fact that whenever $f: M \rightarrow$ $N$ is $K$-quasiregular and $u: N \rightarrow \mathbb{R}$ is $n$-harmonic, then $u \circ f$ is an $\mathcal{A}$-harmonic function, where

$$
\mathcal{A}_{x}(\xi)=\left\langle G_{x} \xi, \xi\right\rangle^{n / 2-1} G_{x} \xi
$$

with

$$
G_{x}= \begin{cases}J(x, f)^{2 / n} T_{x} f^{-1}\left(T_{x} f^{-1}\right)^{*}, & \text { if } J(x, f)>0 \\ \mathrm{id}, & \text { otherwise. }\end{cases}
$$

Here $\left(T_{x} f^{-1}\right)^{*}$ is the transpose of the linear map $T_{x} f^{-1}$. We can choose $\alpha=1 / K$ and $\beta=K^{n-1}$ as the ellipticity constants of $\mathcal{A}$.

\section{A VERSION OF LEWIS' LEMMA}

For our main theorem we need uniform bounds for the growth of an $\mathcal{A}$-harmonic function near its zero set. This calls for a version of Lewis' lemma $\mathrm{L}$. Let us first recall the definition of a Harnack function. Let $M$ be a Riemannian manifold. A function $u: M \rightarrow \mathbb{R}$ is called a Harnack function with constant $\theta$ if

$$
M(h, x, r):=\sup _{B(x, r)} h \leq \theta \inf _{B(x, r)} h
$$

holds in each ball $B(x, r)$ whenever the function $h$ is nonnegative in $B(x, 2 r)$, has the form $h= \pm u+a$ for some $a \in \mathbb{R}$, and $\bar{B}(x, 2 r) \subset M$ is compact. The original version of Lewis' lemma is stated for Harnack functions. It is well known (see [HKM] 6.2]) that $\mathcal{A}$-harmonic functions in the Euclidean setting are Harnack functions with some $\theta$ depending only on $n$ and on the constants $p, \alpha$, and $\beta$ of $\mathcal{A}$. In that case $\theta$ is called the Harnack constant of $\mathcal{A}$. Since we consider $\mathcal{A}$-harmonic functions only on Euclidean domains, we state our lemmata in that context. We write $S^{n-1}(x, r)=\left\{y \in \mathbb{R}^{n}:|y-x|=r\right\}$ for the sphere of center $x$ and radius $r$.

Lemma 2.1. Let $u: \mathbb{R}^{n} \backslash \bar{B}^{n} \rightarrow \mathbb{R}$ be an $\mathcal{A}$-harmonic function such that

$$
\limsup _{|x| \rightarrow \infty} u(x)=+\infty \text { and } \liminf _{|x| \rightarrow \infty} u(x)=-\infty .
$$

Then for every $C_{0}>0$ there exist $R>1$ and $x_{0} \in S^{n-1}(0,100 R)$ such that $u\left(x_{0}\right)=$ 0 and $M\left(u, x_{0}, R\right) \geq C_{0}$.

Proof. For further purposes let us fix a positive integer $k_{0}$ such that if $r>0, x$ and $y$ are points in $S^{n-1}(0, r)$, and $J$ is the shortest arc on $S^{n-1}(0, r)$ connecting the points $x$ and $y$, then there are points $x_{i} \in J, i=1, \ldots, k_{0}$, such that the balls $B^{n}\left(x_{i}, r / 1000\right)$ cover $J$.

Let $C_{0}>0$. By the assumptions on $u$, there exists $r>1$ such that $u(z)=0$ for some $z \in S^{n-1}(0,100 r)$ and

$$
\max _{S^{n-1}(0,100 r)} u \geq \theta^{k_{0}} C_{0}
$$

where $\theta>1$ is the Harnack constant of $\mathcal{A}$. Fix $y \in S^{n-1}(0,100 r)$ such that $u(y)=$ $\max _{S^{n-1}(0,100 r)} u$. We also fix $x \in S^{n-1}(0,100 r)$ such that $u(x)=0$ and $d(x, y)=$ $\operatorname{dist}\left(\{y\}, S^{n-1}(0,100 r) \cap u^{-1}(0)\right)$. Here $d(x, y)$ is the distance of points $x$ and $y$ along the sphere $S^{n-1}(0,100 r)$. The distance from $y$ to the set $S^{n-1}(0,100 r) \cap u^{-1}(0)$ 
is also measured along the sphere. Let $J$ be the shortest arc along $S^{n-1}(0,100 r)$ connecting the points $x$ and $y$. Then $u$ is positive on $J \backslash\{x\}$ by the choice of $x$.

Let us fix points $x_{i} \in J, i=1, \ldots, k_{0}$, such that the balls $B^{n}\left(x_{i}, r / 10\right)$ cover $J$. We arrange points $x_{i}$ in such a way that $y \in B^{n}\left(x_{1}, r / 10\right), x \in B^{n}\left(x_{k_{0}}, r / 10\right)$, and $B^{n}\left(x_{i}, r / 10\right) \cap B^{n}\left(x_{i+1}, r / 10\right) \neq \emptyset$ for all $i$. Let $k$ be the smallest index such that $B^{n}\left(x_{k}, r / 5\right) \cap u^{-1}(0) \neq \emptyset$, and fix a point $z \in B^{n}\left(x_{k}, r / 5\right) \cap u^{-1}(0)$. Since

$$
|z| \geq\left|x_{k}\right|-\left|z-x_{k}\right| \geq 100 r-r / 5>99 r,
$$

we have that $B^{n}\left(x_{k}, r / 5\right) \subset B^{n}(z, 2 r / 5) \subset B^{n}(z,|z| / 100)$. Thus

$$
M(u, z,|z| / 100) \geq M\left(u, x_{k}, r / 5\right) .
$$

Next we show that

$$
M\left(u, x_{k}, r / 5\right) \geq C_{0}
$$

If $k=1$, we have that

$$
M\left(u, x_{1}, r / 5\right) \geq u(y) \geq \theta^{k_{0}} C_{0} \geq C_{0} .
$$

If $k>1$, we may use Harnack's inequality repeatedly in pairs of concentric balls $B^{n}\left(x_{i}, r / 10\right)$ and $B^{n}\left(x_{i}, r / 5\right)$ since $u$ is positive in the set $\bigcup_{i=1}^{k-1} B^{n}\left(x_{i}, r / 5\right)$ and $B^{n}\left(x_{i}, r / 10\right) \cap B^{n}\left(x_{i+1}, r / 10\right) \neq \emptyset$. Hence

$$
M\left(u, x_{k}, r / 5\right) \geq \inf _{B^{n}\left(x_{k-1}, r / 10\right)} u \geq \theta^{1-k} M\left(u, x_{1}, r / 10\right) \geq \theta^{1-k} \theta^{k_{0}} C_{0} \geq C_{0} .
$$

The claim now follows by choosing $x_{0}=z$ and $R=|z| / 100$.

Lemma 2.2 (Lewis' lemma). Let $u: \mathbb{R}^{n} \backslash \bar{B}^{n} \rightarrow \mathbb{R}$ be an $\mathcal{A}$-harmonic function as in Lemma 2.1. Then for every $C_{0}>0$ there exists a ball $B^{n}(a, r)$ such that

(a) $B^{n}(a, 6 r) \subset \mathbb{R}^{n} \backslash \bar{B}^{n}$,

(b) $u(a)=0$, and

(c) $C_{0} \leq M(u, a, 6 r) \leq \theta^{6} M(u, a, r)$,

where $\theta$ is the Harnack constant of $\mathcal{A}$.

Proof. Since the proof of this lemma is almost verbatim to the proof of IM Lemma 19.4.1], we discuss only the modifications needed at the beginning of the proof. Fix $C_{0}>0$, and let $R>1$ and $x_{0} \in S^{n-1}(0,100 R)$ be given by Lemma 2.1. Let $Z=\left\{x \in \mathbb{R}^{n} \backslash \bar{B}^{n}: u(x)=0\right\}$ and $Z_{R}=Z \cap \bar{B}^{n}\left(x_{0}, 41 R\right)$. For each $x \in Z_{R}$ we set

$$
r_{x}=R-\left|x-x_{0}\right| / 41
$$

As in the proof of [M, Lemma 19.4.1] we find a point $a \in Z_{R}$ such that

$$
M\left(u, a, r_{a}\right)=\sup \left\{M\left(u, x, r_{x}\right): x \in Z_{R}\right\} \geq M\left(u, x_{0}, R\right) \geq C_{0} .
$$

Now the point $a$ and radius $r=r_{a}$ satisfy the conditions of the claim. Indeed, conditions (a) and (b) as well as the inequality on the left-hand side of (c) are clearly satisfied. The Harnack type inequality on the right-hand side of (c) can be proved as the corresponding inequality in [IM, Lemma 19.4.1]. 


\section{Big PICARD'S THEOREM}

We say that a Riemannian manifold $M$ is p-parabolic, or, equivalently, has zero p-capacity at infinity if, for each compact set $C \subset M$,

$$
\operatorname{cap}_{p}(M, C):=\inf _{u} \int_{M}|\nabla u|^{p} d m=0
$$

where the infimum is taken over all functions $u \in C_{0}^{\infty}(M)$, with $u \geq 1$ in $C$. Otherwise, we say that $M$ is $p$-hyperbolic.

The following lemma describes the behavior of a quasiregular mapping $f: B^{n} \backslash$ $\{0\} \rightarrow N$ in a neighborhood of 0 assuming that $f$ has an essential singularity at 0 . See [R2, III.2.9, III.2.11] for the case $N=\mathbb{R}^{n}$. Since the proof in our setting involves only minor changes to the proofs in the Euclidean case, we just sketch the idea.

Lemma 3.1. Let $N$ be a Riemannian n-manifold and $f: B^{n} \backslash\{0\} \rightarrow N$ a quasiregular mapping having an essential singularity at the origin. Then $f\left(B^{n}(0, r) \backslash\{0\}\right)$ is n-parabolic for every $r \in(0,1)$.

Proof. Suppose on the contrary that $f\left(B^{n}\left(0, r_{0}\right) \backslash\{0\}\right)$ is $n$-hyperbolic for some $r_{0} \in(0,1)$. Since $f$ has an essential singularity at the origin, we find a compact set $C \subset N$ such that $f\left(B^{n}(0, r) \backslash\{0\}\right) \cap C \neq \emptyset$ for every $r \in(0,1)$. Hence we may choose a sequence $\left(x_{k}\right)$ of points in $B^{n}\left(0, r_{0}\right) \backslash\{0\}$ tending to the origin such that $f\left(x_{k}\right) \rightarrow z$ for some $z \in C$. Let $\left(y_{k}\right)$ be another sequence in $B^{n}\left(0, r_{0}\right) \backslash\{0\}$ tending to the origin. We show that $f\left(y_{k}\right) \rightarrow z$, too. If this is not the case, we may assume that $d\left(f\left(x_{k}\right), f\left(y_{k}\right)\right) \geq \delta>0$ for all $k$. Let $F_{k}$ be a continuum connecting $x_{k}$ and $y_{k}$ in $\bar{B}^{n}\left(0, \max \left\{\left|x_{k}\right|,\left|y_{k}\right|\right\}\right) \backslash\{0\}$. Then

$$
\operatorname{cap}_{n}\left(f\left(B^{n}\left(0, r_{0}\right) \backslash\{0\}\right), f\left(F_{k}\right)\right) \leq K_{I}(f) \operatorname{cap}_{n}\left(B^{n}\left(0, r_{0}\right) \backslash\{0\}, F_{k}\right)
$$

by the $K_{I}$-inequality (see [R2 II.10.10]). Here $K_{I}(f)$ is the inner dilatation of $f$. On the other hand,

$$
\operatorname{cap}_{n}\left(B^{n}\left(0, r_{0}\right) \backslash\{0\}, F_{k}\right) \rightarrow 0
$$

as $k \rightarrow \infty$. However, there exists $s>0$ such that $f\left(F_{k}\right)$ connects $B(z, s)$ and $N \backslash B(z, 4 s)$ for every sufficiently large $k$ and such that $B(z, 4 s)$ is contained in a bilipschitz chart. Hence there exists a constant $c>0$, independent of $k$, such that, for all sufficiently large $k$,

$$
\operatorname{cap}_{n}\left(f\left(B^{n}\left(0, r_{0}\right) \backslash\{0\}\right), f\left(F_{k}\right)\right) \geq c>0
$$

by the assumption that $f\left(B^{n}\left(0, r_{0}\right) \backslash\{0\}\right)$ be $n$-hyperbolic. This contradicts (3.1) and (3.2). Hence $f\left(y_{k}\right) \rightarrow z$, but this contradicts the essential singularity assumption on $f$. Therefore $f\left(B^{n}(0, r) \backslash\{0\}\right)$ is $n$-parabolic for every $r \in(0,1)$.

The following lemma is originally given in HR1]. See also the remark after [HR3. Lemma 3.2].

Lemma 3.2 ([HR1],[HR3]). Let $N$ be an n-parabolic Riemannian manifold. Suppose that $C \subset N$ is compact such that $N$ has $q$ ends $V_{1}, \ldots, V_{q}$ with respect to $C$. Then there exist $n$-harmonic functions $v_{j}, j=2, \ldots, q$, and a positive constant $\kappa$ 
such that

$$
\begin{aligned}
& \left|v_{j}\right| \leq \kappa \text { in } C, \\
& \left|v_{j}-v_{i}\right| \leq 2 \kappa \text { in } V_{1}, \\
& \sup _{V_{1}} v_{j}=\infty, \\
& \inf _{V_{j}} v_{j}=-\infty, \\
& v_{j} \text { is bounded in } V_{k} \text { for } k \neq 1, j, \\
& \text { if } v_{j}(x)>\kappa, \text { then } x \in V_{1}, \\
& \text { if } v_{j}(x)<-\kappa, \text { then } x \in V_{j} .
\end{aligned}
$$

Proof of Theorem 1.3. The proof is similar to that of Theorem 1.4 given in [HR3]. However, for the reader's convenience we give all the details. Suppose that there exists a $K$-quasiregular mapping $f: B^{n} \backslash\{0\} \rightarrow N$ having an essential singularity at the origin. Let $C \subset N$ be a compact set such that $N$ has $q$ ends $V_{1}, \ldots, V_{q}$ with respect to $C$. For every $j=2, \ldots, q$ let us fix an $n$-harmonic function $v_{j}$ with properties (3.3) - (3.9) given in Lemma [3.2. For every $j=2, \ldots, q$ we set $u_{j}=v_{j} \circ f \circ \sigma$, where $\sigma$ is a Möbius transformation such that $\sigma\left(\mathbb{R}^{n} \backslash \bar{B}^{n}\right)=B^{n} \backslash\{0\}$. Thus functions $u_{j}$ are $\mathcal{A}$-harmonic in $\mathbb{R}^{n} \backslash \bar{B}^{n}$. Next we show that they satisfy the assumptions of Lemma 2.1 that is,

$$
\limsup _{|x| \rightarrow \infty} u_{j}(x)=+\infty \text { and } \liminf _{|x| \rightarrow \infty} u_{j}(x)=-\infty
$$

This can be seen by observing that the sets $\left\{x \in N: v_{j}(x)>c\right\}$ and $\{x \in$ $\left.N: v_{j}(x)<-c\right\}$ are nonempty and open for every $c>0$ and $j=2, \ldots, q$. By Lemma 3.1, $f\left(B^{n}(0, r) \backslash\{0\}\right)$ intersects these sets for every $r \in(0,1)$, and therefore (3.10) follows. By Lemma 2.2 there are sequences $\left(x_{i}\right)$ and $\left(r_{i}\right)$ of $\mathbb{R}^{n} \backslash \bar{B}^{n}$ and $(0, \infty)$, respectively, such that $u_{2}\left(x_{i}\right)=0, B^{n}\left(x_{i}, 3 r_{i}\right) \subset \mathbb{R}^{n} \backslash \bar{B}^{n}$,

$$
M\left(u_{2}, x_{i}, 3 r_{i}\right) \leq \theta^{6} M\left(u_{2}, x_{i}, r_{i} / 2\right),
$$

and $M\left(u_{2}, x_{i}, r_{i} / 2\right) \rightarrow \infty$ as $i \rightarrow \infty$. Let us fix an index $i$ such that $M\left(u_{2}, x_{i}, r_{i} / 2\right) \geq$ $4 \theta \kappa$, where $\theta>1$ is the Harnack constant of $\mathcal{A}$ and $\kappa$ is the constant in Lemma 3.2. We write $x=x_{i}$ and $r=r_{i}$. By (3.8), $(f \circ \sigma)\left(B^{n}(x, r / 2)\right) \cap V_{1} \neq \emptyset$. Thus, by (3.4), we have

$$
M\left(u_{2}, x, s\right)-2 \kappa \leq M\left(u_{j}, x, s\right) \leq M\left(u_{2}, x, s\right)+2 \kappa
$$

whenever $s \geq r / 2$. Next we conclude by using Harnack's inequality that

$$
M\left(u_{j}, x, r\right) \leq(\theta-1) M\left(-u_{j}, x, 2 r\right)
$$

for all $j$. Let us first show that $u_{j}(z)=0$ for some $z \in B^{n}(x, r)$. Suppose on the contrary that $u_{j}>0$ in $B^{n}(x, r)$. Then $u_{j}(y) \leq \theta u_{j}(x)$ for all $y \in B^{n}(x, r / 2)$ by Harnack's inequality. Since $M\left(u_{2}, x, r / 2\right) \geq 4 \theta \kappa$, there exists $y \in B^{n}(x, r / 2)$ such that $u_{j}(y)>2 \theta \kappa$ by (3.11). Thus $u_{j}(x)>2 \kappa$, and so $x \in V_{1}$. By (3.4), $u_{2}(x) \geq u_{j}(x)-2 \kappa>0$, contradicting the assumption $u_{2}(x)=0$. Therefore there exists $z \in B^{n}(x, r)$ such that $u_{j}(z)=0$. Thus $\inf _{B^{n}(x, r)} u_{j} \leq 0$. Inequality (3.12) 
follows now from the calculation

$$
\begin{aligned}
M\left(u_{j}, x, r\right) & =\sup _{B^{n}(x, r)} u_{j}=\sup _{B^{n}(x, r)}\left(u_{j}-\inf _{B^{n}(x, 2 r)} u_{j}\right)+\inf _{B^{n}(x, 2 r)} u_{j} \\
& \leq \theta \inf _{B^{n}(x, r)}\left(u_{j}-\inf _{B^{n}(x, 2 r)} u_{j}\right)+\inf _{B^{n}(x, 2 r)} u_{j} \\
& =\theta \inf _{B^{n}(x, r)} u_{j}+(1-\theta) \inf _{B^{n}(x, 2 r)} u_{j} \\
& \leq-(\theta-1) \inf _{B^{n}(x, 2 r)} u_{j}=(\theta-1) \sup _{B^{n}(x, 2 r)}\left(-u_{j}\right) \\
& =(\theta-1) M\left(-u_{j}, x, 2 r\right),
\end{aligned}
$$

since $u_{j}-\inf _{B^{n}(x, 2 r)} u_{j} \geq 0$ in $B^{n}(x, 2 r)$.

Inequalities (3.11) and (3.12), and the assumption $M\left(u_{2}, x, r / 2\right) \geq 4 \theta \kappa$ together yield the inequality

$$
M\left(u_{2}, x, r\right) \leq \theta M\left(-u_{j}, x, 2 r\right)
$$

Indeed,

$$
\begin{aligned}
M\left(u_{2}, x, r\right) & \leq M\left(u_{j}, x, r\right)+\theta^{-1} M\left(u_{2}, x, r\right) \\
& \leq(\theta-1) M\left(-u_{j}, x, 2 r\right)+\theta^{-1} M\left(u_{2}, x, r\right),
\end{aligned}
$$

which is equivalent to (3.13). We fix $z_{j} \in \bar{B}^{n}(x, 2 r)$ such that

$$
M\left(-u_{j}, x, 2 r\right)=-u_{j}\left(z_{j}\right) .
$$

The well-known oscillation estimate (see e.g. [HKM, 6.6])

$$
\underset{B^{n}(y, \rho)}{\operatorname{Osc}} u_{j} \leq c(\rho / r)^{\gamma} \underset{B^{n}(y, r)}{\operatorname{osc}} u_{j}
$$

together with [HR1, Lemma 4.2] and (3.11) imply that

$$
\underset{B^{n}\left(z_{j}, \rho\right)}{\operatorname{osc}} u_{j} \leq c_{1}(\rho / r)^{\gamma} M\left(u_{2}, x, 3 r\right)
$$

for $\rho \in(0, r)$. See [HR1, (5.5)] for details. Here $c, c_{1}$, and $\gamma$ depend only on $n$ and $K$, since $\theta=\theta(n, K)$. Thus

$$
\begin{aligned}
\max _{\bar{B}^{n}\left(z_{j}, \rho\right)} u_{j} & =\underset{B^{n}\left(z_{j}, \rho\right)}{\operatorname{osc}} u_{j}+\min _{\bar{B}^{n}\left(z_{j}, \rho\right)} u_{j} \\
& \leq c_{1}(\rho / r)^{\gamma} M\left(u_{2}, x, 3 r\right)+u_{j}\left(z_{j}\right) \\
& \leq c_{1}(\rho / r)^{\gamma} M\left(u_{2}, x, 3 r\right)-\theta^{-1} M\left(u_{2}, x, r\right)
\end{aligned}
$$

by (3.15), (3.14), and (3.13). Since $M\left(u_{2}, x, 3 r\right) \leq \theta^{6} M\left(u_{2}, x, r\right)$, we obtain

$$
c_{2}(\rho / r)^{\gamma} M\left(u_{2}, x, 3 r\right) \leq(2 \theta)^{-1} M\left(u_{2}, x, r\right)
$$

by choosing $\rho=\left(2 \theta^{7} c_{2}\right)^{-1 / \gamma} r$. Hence

$$
\max _{\bar{B}^{n}\left(z_{j}, \rho\right)} u_{j} \leq-(2 \theta)^{-1} M\left(u_{2}, x, r\right) \leq-2 \kappa .
$$

By (3.9), we conclude that $(f \circ \sigma)\left(B^{n}\left(z_{j}, \rho\right)\right) \subset V_{j}$ and hence the balls $B^{n}\left(z_{j}, \rho\right)$ are disjoint. Since $B^{n}\left(z_{j}, \rho\right) \subset B^{n}(x, 3 r)$, there can be at most $(3 r / \rho)^{n}$ of them. Hence $q \leq(3 r / \rho)^{n}$, which is an upper bound that depends only on $n$ and $K$. 


\section{REFERENCES}

[A] L. V. Ahlfors, Conformal invariants: topics in geometric function theory, McGraw-Hill Series in Higher Mathematics, McGraw-Hill Book Co., New York, 1973. MR50:10211

[EL] A. Eremenko and J. L. Lewis, Uniform limits of certain A-harmonic functions with applications to quasiregular mappings, Ann. Acad. Sci. Fenn. Ser. A I Math. 16 (1991), 361-375. MR.93b:35039

[IM] T. Iwaniec and G. Martin, Geometric Function Theory and Non-linear Analysis, Oxford Mathematical Monographs, Clarendon Press, Oxford, 2001. MR2003c:30001

$[$ HKM] J. Heinonen, T. Kilpeläinen, and O. Martio, Nonlinear Potential Theory of Degenerate Elliptic Equations, Oxford Mathematical Monographs, Clarendon Press, Oxford - New York - Tokyo, 1993. MR 94e:31003

[HR1] I. Holopainen and S. Rickman, A Picard type theorem for quasiregular mappings of $\mathbb{R}^{n}$ into n-manifolds with many ends, Rev. Mat. Iberoamericana 8 (1992), 131-148. MR.94g:30021

[HR2] I. Holopainen and S. Rickman, Quasiregular mappings of the Heisenberg group, Math. Ann. 294 (1992), 625-643. MR93m:30031

[HR3] I. Holopainen and S. Rickman, Ricci curvature, Harnack functions, and Picard type theorems for quasiregular mappings, Analysis and Topology, World Sci. Publishing, River Edge, NJ, 1998, 315-326. MP 99j:30026

[L] J. L. Lewis, Picard's theorem and Rickman's theorem by way of Harnack's inequality, Proc. Amer. Math. Soc. 122 (1994), 199-206. MR.94k:30052

[R1] S. Rickman, On the number of omitted values of entire quasiregular mappings, J. Analyse Math. 37 (1980), 100-117. MR81m:30030

[R2] S. Rickman, Quasiregular mappings, Ergebnisse der Mathematik und ihrer Grenzgebiete 26, Springer-Verlag, Berlin - Heidelberg - New York, 1993. MR95g:30026

Department of Mathematics, P.O. Box 4 (Yliopistonkatu 5), Fin-00014, University of Helsinki, Finland

E-mail address: ilkka.holopainen@helsinki.fi

Department of Mathematics, P.O. Box 4 (Yliopistonkatu 5), Fin-00014, University of Helsinki, Finland

E-mail address: pekka.pankka@helsinki.fi 